\title{
Epistemologia e difusão do conhecimento: uso de pesquisa aplicação na educação tecnológica e STEAM - processo de formação de docentes para a Educação de Jovens e Adultos do SESI, Bahia
}

\author{
Gisele Marcia de Oliveira Freitas ${ }^{1}$ \\ Francisca de Paula Santos da Silva ${ }^{2}$ \\ Alfredo Eurico Rodrigues Matta ${ }^{3}$
}

Resumo: Este artigo trata sobre a base epistemológica que favorece o uso da Pesquisa Aplicação em contexto de formação de docentes para Educação de Jovens e Adultos no SESI Bahia. Esta pesquisa, em andamento, vem adotando a educação tecnológica e STEAM na prática pedagógica de ensino de arte, ciência, tecnologia, engenharia e matemática, agregando abordagem e metodologias participativas, no caso a Pesquisa Aplicação, ou Design Based-Research (DBR). Trata-se de uma abordagem metodológica peculiar, inovadora e de natureza aplicada, que possibilita uma interação e colaboração com a realidade dos sujeitos constituintes do ambiente. O referencial teórico adotado inclui, entre outros, autores como: Castells (2011); Matta, Silva e Boaventura (2014) e Vygotsky (1998; 2001; 2004; 2007). Os resultados apontam para uma pesquisa de cunho epistemológico fundamentado no socioconstrutivismo e amparada pela metodologia de Pesquisa Aplicação, que é uma abordagem de investigação inovadora por reunir as vantagens das metodologias qualitativas e das quantitativas.

Palavras-chave: Epistemologia. Socioconstrutivismo. Formação docente. Pesquisa Aplicação.

\begin{abstract}
This article discusses the epistemological basis that favors the use of Application Research in context of teacher training for Youth and Adult Education, in SESI Bahia. In progress, the research adopts technological education and STEAM in the pedagogical practice of teaching art, science, technology, engineering and mathematics, bringing together participatives approach and methodologies, in this case, the Application Research, or Design-Based Research (DBR). It is a peculiar, innovative and applied methodology that enables interaction and collaboration with the reality of the constituent subjects of the environment. The theoretical reference includes authors such as Castells (2011); Matta, Silva and Boaventura (2014) and Vygotsky (1998; 2001; 2004; 2007), among others. The results point to an epistemological study, based on socio-constructivism and the methodology of Application Research. This methodology is an innovative research approach that brings together the advantages of qualitative and quantitative methodologies.
\end{abstract}

Keywords: Epistemology. Teacher training. Socio-constructivism. Research Application.

\footnotetext{
${ }^{1}$ Doutoranda em Difusão do Conhecimento pela Universidade Federal da Bahia. Mestre em Educação de Jovens e Adultos pela Universidade do Estado da Bahia (UNEB). Mestre em Formación de Profesores de Español pela Universidad de Alcalá (UAH). Mestre em Comunicación y Educación en la Red pela Universidad Nacional de Educación a Distancia (UNED). E-mail: adelantegisele@yahoo.com.br.

2 Pós-Doutoramento em Educação, Universidade de Coimbra, Portugal. Doutoramento em Educação, Universidade Federal da Bahia (UFBA). Mestrado em Administração (UFBA). Especialização em Administração, Pontifícia Universidade Católica de Minas Gerais, e em Psicopedagogia Junguiana, Instituto Junguiano da Bahia. E-mail: fcapaula@gmail.com.

3 Pesquisador do CNPQ, DT2. Mestrado em História pela Universidade Federal da Bahia (UFBA). Doutorado em Educação pela (UFBA)/ Université Laval (Canadá). Pós-Doutorado na Universidade do Porto em Educação a Distância e Comunidades de Aprendizagem Internacionais em Língua Portuguesa, apoiado pelo CNPQ. E-mail: alfredomatta@gmail.com.
} 


\section{Introdução}

A tecnologia digital entrou por todo o interstício da vida social. $\mathrm{Na}$ contemporaneidade, vivenciamos a codificação digital e articulação em rede o que promove novas possibilidades de fazer, relacionar-se, produzir e criar, o que não se imaginava em um contexto analógico (BONILLA; PRETTO, 2015).

A mudança da tecnologia analógica e eletromecânica para a tecnologia digital caracteriza-se como a Terceira Revolução Industrial (RIFKIN, 2011). Não se trata apenas de uma revolução industrial, mas cultural e comunicativa, gerando a sociedade em rede (CASTELLS, 2011), a cidadania digital (RIBBLE; BAILEY; ROSS, 2014) os prosumer (produtores-consumidores na rede) (JENKINS, 2007; DE PABLOS, 2015) e, em geral, a era digital (CASTELLS, 2012).

O projeto de pesquisa em andamento propõe como solução a qualificação dos docentes da educação de jovens e adultos do SESI Bahia para a prática de soluções inovadoras, criativas e consistentes por meio das metodologias ativas e Science, Technology, Engineering, the Arts and Mathematics (STEAM, Ciência, Tecnologia, Engenharia, Arte e Matemática).

A metodologia STEAM estimula o desenvolvimento de competências e habilidades, a exemplo do pensamento crítico, resolução de problemas, empreendedorismo, trabalho colaborativo, engenhosidade e compreensão dos fundamentos científicos, por meio do protagonismo, da relação teoria com a prática e do desenvolvimento do perfil pesquisador, investigativo, crítico e autônomo, capaz de utilizar o conhecimento para realizar mudanças e transformações no processo de aprendizado.

A terminologia STEAM nasce em 2006, fruto do trabalho do Georgette Yakman (2015), e se apresenta como uma metodologia que estuda a Ciência e Tecnologia interpretada por meio da Engenharia e das Artes. É um marco na aprendizagem, porque com base em problemas identificados pela comunidade, a vontade de pesquisar e a curiosidade se convertem em motor e guia do conhecimento, um ponto de partida para a aprendizagem significativa (CILLERUELO; ZUBIAGA, 2014).

Tal metodologia promove uma aproximação interdisciplinar integrada, conectada com o mundo real, e dirigida à resolução de problemas (PBL). A relação entre arte, ciência e tecnologia permite o desenvolvimento de conexões curriculares estabelecendo relações entre competências e os temas do currículo (CILLERUELO; ZUBIAGA, 2014).

As metodologias ativas estimulam o engajamento e o compromisso com a própria aprendizagem, estão amparadas pelos meios e recursos tecnológicos. Para Moran (2015, p. 18), “as metodologias ativas são pontos de partida para avançar para processos mais avançados de reflexão, de integração cognitiva, de generalização, de reelaboração de novas práticas". 
A proposta de pesquisa dialoga com os Objetivos de Desenvolvimento Sustentável (ODS), os quais apresentam a necessidade de o ser humano refletir e transformar seu modo de pensar e agir, exercendo uma postura ética e consciente da coletividade para a manutenção da vida no planeta.

O ODS 4 - Educação de Qualidade possui entre seus descritores a seguinte meta: até 2030, os países deverão substancialmente aumentar o contingente de professores qualificados, os alunos devem adquirir conhecimento e habilidades necessárias para promover o desenvolvimento sustentável e práticas de empreendedorismo.

É nessa vertente que a proposta de pesquisa aplicação busca preparar os docentes para o desafio de proporcionar aos estudantes da educação de jovens e adultos a preparação para a vida nas dimensões da cidadania, empreendedorismo, desenvolvimento sustentável e mundo do trabalho.

Baseado nessa reflexão introdutória, na breve descrição da pesquisa aplicação que se pretende realizar, o artigo tem o objetivo de apresentar as bases epistemológicas, as primeiras definições, bem como encaminhamentos possíveis, levantamentos e análises que sustentarão a pesquisa aplicada em andamento.

Situada no âmbito das Epistemologias para a Difusão do Conhecimento, esta produção constitui uma discussão teórico-analítica, de caráter qualitativo (LÜDKE; ANDRÉ, 1986), instrumentalizada por uma pesquisa bibliográfica.

Para tanto, o trabalho organiza-se em 5 (cinco) seções. A introdutória, que apresenta os pressupostos básicos do trabalho, os objetivos do artigo e os procedimentos metodológicos utilizados nesta pesquisa. Em seguida estão o contexto e o referencial teórico que subsidiou os conceitos de referência. Logo após, a apresentação e a discussão dos resultados que orientam as considerações finais do artigo, as quais destacam as possibilidades de inovações e caminhos possíveis de serem seguidos.

\section{O contexto da pesquisa: um caminhar no Sesi Bahia e em seu entorno}

O lócus da investigação científica será a Escola SESI Reitor Miguel Calmon, situada no Retiro, na cidade de Salvador, Bahia, na região do Antigo Quilombo do Cabula. Essa região abarca 17 (dezessete) bairros da cidade de Salvador, a saber: Arenoso; Beiru/Tancredo Neves; Cabula; Doron; Engomadeira; Estrada das Barreiras; Fazenda Grande do Retiro; São Gonçalo do Retiro; Arraial do Retiro; Pernambués; Saramandaia; Resgate; Mata Escura; Novo Horizonte; Narandiba; Saboeiro e Sussuarana, conforme detalha a figura 01 . 
Figura 01 - Mapa dos Bairros do Turismo de Base Comunitária no Cabula (TBC) e Entorno.

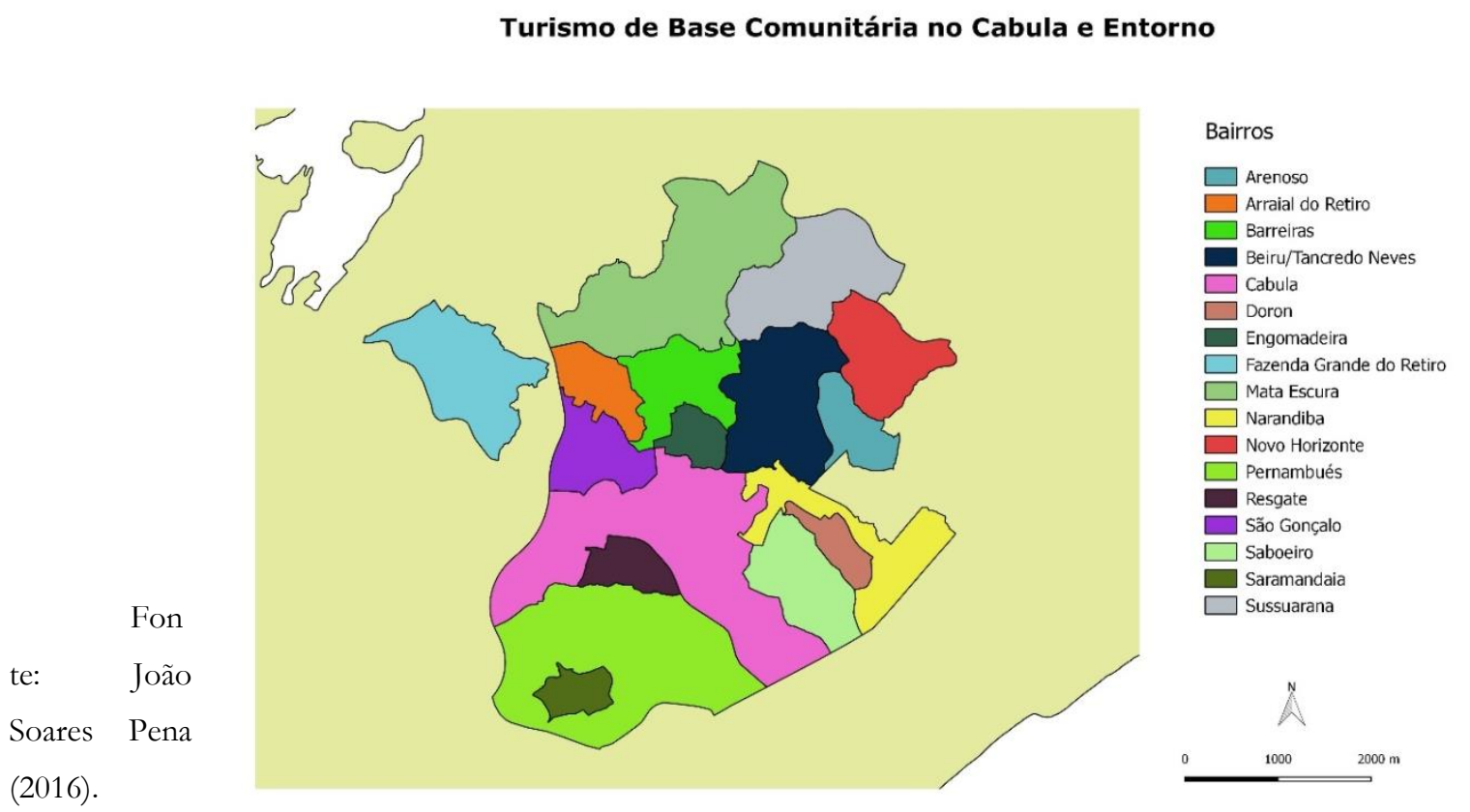

O Serviço Social da Indústria (SESI) nasce em um contexto político e social bem conturbados, marcados por conflitos em vários países, período pós Segunda Guerra Mundial. Nesse cenário, o Brasil vivia dificuldades políticas e necessitava preparar os trabalhadores para as mudanças introduzidas pela recente industrialização. É nesse contexto brasileiro e internacional que o Presidente Eurico Gaspar Dutra assina, em 1946, o Decreto Lei no 9.403, que atribui à Confederação Nacional da Indústria (CNI) a tarefa de criar, organizar e dirigir o SESI (FREITAS, 2018).

A Educação de Jovens e Adultos (EJA) é uma das ações estratégicas do SESI para atender à demanda da população e da indústria que não concluíram seus estudos na idade regular. A atuação da EJA no SESI Bahia começou nos anos noventa, quando foram implantadas as primeiras salas de aulas na região metropolitana de Salvador e Litoral Norte (FREITAS, 2018).

Ao longo de vinte e três anos, o SESI Bahia já realizou mais de 130 mil matrículas nessa modalidade de ensino. No ano passado, em 2018, foram realizadas 8.675 matrículas, acalcando a $4^{\mathrm{a}}$ posição, em relação ao número de matrículas na EJA dentro da Rede SESI de Educação (SESI, 2018).

Com a problemática, objetivos apresentados e o lócus da pesquisa devidamente contextualizado, nasce este artigo, cujo propósito é mostrar reflexões epistemológicas que constituem as bases para a construção da pesquisa aplicada a ser realizada no âmbito do programa de Doutorado em Difusão do Conhecimento. 


\section{As bases epistemológicas da pesquisa aplicada: uma visão sob a ótica de Vygotsky}

A epistemologia está intrinsecamente relacionada às assunções filosóficas da teoria do conhecimento, permite articular técnicas, métodos e teorias em uma determinada concepção de ciência. A concepção epistemológica a ser adotada na pesquisa aplicação em andamento é a socioconstrutivista.

Representa um grande desafio associar comunidade, inovação e socioconstrutivismo em uma única abordagem de pesquisa. A inovação, nesse caso, não visa o abandono de práticas pedagógicas atuais, em substituição de novas abordagens, mas sim a socialização de novos saberes e fazeres coletivos que dialoguem em benefício da comunidade e coletivo do TBC. Nesse sentido, a metodologia da pesquisa aplicação será utilizada para subsidiar a inovação que se pretende construir, a comunidade se situa como a principal parceira desse diálogo.

A epistemologia socioconstrutivista resulta em uma bricolagem do pensamento, é um campo de dúvida e incertezas. É nessa incerteza que deixa de ser epistemologia e vira método de pesquisa, deixando as dúvidas no ar e definindo em termos práticos o que se pretende realizar.

A epistemologia Socioconstrutivista possui uma interdependência entre pensamento e linguagem, um pressuposto diferente dos construtivistas ou neuro construtivistas, que consideram o pensamento algo diferente da linguagem. Tal abordagem tampouco considera a linguagem igual ao pensamento como os pós-estruturalistas. Para os Socioconstrutivistas, o pensamento tangencia a linguagem e esta é um elemento mediador de práxis, pois na sua concepção não é na mente que se constrói o conhecimento.

O conhecimento é construído na práxis, à medida que é vivenciado pelo coletivo. A verdade para os epistemólogos Socioconstrutivistas é o encontro da práxis dos envolvidos, algo compartilhado, dinâmico, contextualizado e transitório. Não é imposta, ela é construída à proporção que é vivida e experienciada pelos envolvidos.

A pesquisa em andamento estará amparada na teoria socioconstrutivista tendo como princípio norteador os estudos de Vygotsky. Para o autor, o indivíduo é o resultado do processo histórico e social em que a linguagem desempenha um papel essencial, o conhecimento é um processo de interação do sujeito com o meio social e cultural. Para o autor, atividades cognitivas, como memória, percepções e os pensamentos, são características fundamentais do ser humano, resultado de uma aprendizagem sociocultural.

Vygotsky (2007) propõe que o sujeito atue sobre a realidade para adaptar-se a ela transformando-a e transformando-se a si mesmo por meios dos instrumentos psicológicos chamados de mediadores, utilizando-se de ferramentas, que são os recursos materiais e de signos, que é a linguagem. Para o autor, é por meio da interação do pensamento com a linguagem que se produzem mudanças radicais na cognição humana. 
Diante do fundamento epistemológico apresentado, a pesquisa de doutorado será desenvolvida sobre o amparo metodológico da Desing-Based Research (DBR), também conhecida como Pesquisa Aplicação. A metodologia de pesquisa contribuirá com a formação de professores da EJA do SESI Bahia para a prática de soluções inovadoras, criativas e consistentes por meio das metodologias ativas e Science, Technology, Engineering, the Arts and Mathematics (STEAM, Ciência, Tecnologia, Engenharia, Arte e Matemática).

A proposta de pesquisa aplicação será construída sobre os fundamentos de Vygotsky (2007), que considera a atividade humana um conjunto de ações culturalmente determinadas e contextualizadas, realizadas em cooperação com outros.

Sobre o termo cooperação, a metodologia de pesquisa adotada, a DBR, é desenvolvida com esse propósito, promover a formação docente, dentro de uma perspectiva colaborativa de formação entre pares sobre o uso das tecnologias em práticas docentes.

A formação docente estará amparada no tripé sujeito, objeto de conhecimento e os artefatos ou instrumentos socioculturais digitais amplamente influenciados pelo contexto cultural dos sujeitos envolvidos. O contexto cultural, nesse sentido, desempenha um papel essencial e determinante do desenvolvimento do sujeito, que não receberá passivamente a formação, mas atuará na constante troca e retroalimentação, tomando como ponto de partida o contexto social em que está inserido.

Para Vygotsky (2007), o sujeito, embora protagonista de seu papel, não é a única variável na aprendizagem. Sua história de vida, condição social, relações sociais, conjuntura histórica, recursos disponíveis são variáveis que não só apoiam a aprendizagem, mas também são parte integral dela.

Diante disso, a pesquisa de Doutorado busca na formação docente a contribuição para a promoção de práticas pedagógicas que fomentem o uso das tecnologias da informação e comunicação no cotidiano dos sujeitos da EJA.

Na concepção de Vygotsky (2004), o processo de desenvolvimento cognitivo individual não é independente ou autônomo dos contextos socioculturais. Segundo o autor, não é possível pôr em prática nenhum processo de aprendizagem sem considerar o contexto histórico-cultural em que estão imersos os sujeitos, os quais trazem consigo uma série de instrumentos e práticas sociais historicamente determinadas e organizadas.

\section{Apresentação e discussão dos resultados: as dimensões filosóficas e epistemológicas da pesquisa aplicação}

Mergulhando nas dimensões epistemológicas da pesquisa, encontramos 5 (cinco) grandes dimensões: SESI, TBC, inovação, DBR e Socioconstrutivismo. Na análise dessas dimensões, o caminhar 
nos leva à complexidade. Para Morin (2005, p. 35), “a complexidade não compreende apenas quantidades de unidade e interações que desafiam nossas possibilidades de cálculo: ela compreende também incertezas, indeterminações, fenômenos aleatórios". Desse modo, declara Morin (2005, p.06), a complexidade surge na falha da simplicidade, mas "integra tudo aquilo que põe ordem, clareza, distinção precisão no conhecimento".

Aprofundando na dimensão DBR, Matta, Silva e Boaventura (2014) afirmam que a DBR é também conhecida como Pesquisa Aplicação, compondo uma abordagem de investigação inovadora que reúne as vantagens das metodologias tanto qualitativas quanto quantitativas. Como destaque, apresenta a intenção da superação da dicotomia entre pesquisa qualitativa e quantitativa, pois possui o objetivo de desenvolvimento de pesquisas que busquem "soluções práticas e inovadoras para os graves problemas da educação" (MATTA; SILVA; BOAVENTURA, 2014, p. 25).

É uma abordagem metodológica peculiar, inovadora e de natureza aplicada, que possibilita uma interação, a colaboração com a realidade dos sujeitos constituintes do ambiente. Para Santiago e Matta (2016, p.3) "[...] a metodologia DBR foca no desenvolvimento de aplicações e soluções práticas, em particular as digitais, nas quais os Ambientes Virtuais de Aprendizagem são suportados".

Assim sendo, a abordagem metodológica desta pesquisa viabilizará a tessitura da significação percebida e empregada pelos sujeitos, nas dinâmicas das relações socioculturais na condição de educadores e protagonistas do seu processo de formação.

A epistemologia Socioconstrutivista possibilitará uma construção científica amparada nas particularidades do contexto da pesquisa, em que os sujeitos e as relações socioculturais se convertem na própria origem da fonte do saber. Situar-se nesse lugar lhes permite refletir sobre as coisas que sabem e podem compartilhar, e refletir sobre o que necessita aprimorar, seus interesses e curiosidades de acordo com o seu contexto social de atuação profissional.

Nessa perspectiva, a pesquisa ganha sentido graças à sua natureza aplicada, em que se elaborarão, no coletivo, roteiros de itinerários formativos em tecnologias da informação e comunicação Esses roteiros serão construídos por meio de ciclos interativos que se pretende implementar com os sujeitos da pesquisa, contribuindo assim para a compreensão e construção do conhecimento compartilhado.

Na qualidade de pesquisadora, o papel a ser assumido é de ator social, com um olhar qualitativo sobre o fenômeno para a captação das dimensões que esta pesquisa objetiva. Assim, as apreensões, os sentidos, significados, as ações, os processos formativos e contribuições da pesquisa na formação docente serão construídos no coletivo com os atores sociais que comporão a pesquisa de Doutorado.

\section{Considerações finais}


Considerando que a pesquisa em questão está em fase inicial do Doutorado, evidencia-se como grande desafio pôr em prática uma pesquisa aplicada, envolvendo sujeitos formadores que estarão em processo de formação, o que implica a possibilidade de um possível "fracasso" ou não alcance dos objetivos do projeto. Entretanto, espera-se que o amparo epistemológico e metodológico, nesse caso, respectivamente o socioconstrutivismo como fundamento epistemológico e a metodologia de pesquisa DBR, se possa empreender uma pesquisa aplicação que não almeje a completude da investigação, mas fomentar a continuidade da formação nos sujeitos, consequentemente, novos objetos de pesquisa.

Para a significação dos processos tecnológicos, a formação docente não pode separar o recurso do sujeito, uma vez que o potencial do artefato é o uso consciente dos sujeitos, os seres socialmente culturais. A formação do educador precisa ter significação, subjetivação e sustentação social para a implantação de uma cultura digital no cotidiano escolar que possibilite aos alunos o uso das tecnologias digitais da comunicação e informação de forma crítica, significativa, reflexiva e ética para comunicar-se, acessar e disseminar informações, produzir conhecimentos, resolver problemas, elaborar propostas, construir argumentações, entre outras práticas em que os sujeitos desenvolvam saberes.

Os desafios e novos caminhos da pesquisa permearão na construção coletiva de itinerários formativos em educação tecnológica, competência digital e STEAM, contribuindo para a aprendizagem permanente dos educadores atuantes na educação básica de jovens e adultos do SESI Bahia e na realização de ciclos de formação docentes por meio da DBR ou Pesquisa-Aplicação.

Diante desses desafios, conclui-se que a epistemologia socioconstrutivista, amparada pela metodologia de pesquisa DBR, buscará por meio de ações coletivas e colaborativas a sustentação da pesquisa aplicada.

\section{Referências}

BONILLA, M. H. S.; PRETTO, N. L. Política educativa e cultura digital: entre práticas escolares e práticas sociais. Perspectiva (UFSC), v. 33, p. 499-521, 2015.

CASTELLS, M. La era de la información: economía, sociedad y cultura. Vol. 1. Madrid: Alianza, 2011.

Rede de indignación y esperanza: los movimientos sociales en la era de Internet. Madrid: Alianza D. L, 2012. 
CILLERUELO L.; ZUBIAGA A. Una aproximación a la Educación STEAM. Prácticas educativas en la encrucijada arte, ciencia y tecnología. Jornadas de Psicodidáctica, 2014. Disponível em: <https://www.augustozubiaga.com/web/wp-content/uploads/2014/11/STEM-TO-STEAM.pdf>.

Acesso em: 25 de mai. de 2019.

FREITAS, G. M. O.; RIBEIRO, T.; AMORIM, A.; FREITAS, K. S. A prática da gestão da educação de jovens e adultos no serviço social da indústria no estado da Bahia. Educação em Revista (online), v. 34, p. 1-28, 2018a.

FREITAS, G. M. O.; MORAIS, C. L.; EVANGELISTA. P.; SILVA. S. Educação de Jovens e Adultos Profissionalizante a distância: experiência pedagógica do SESI e Senai Bahia. In: Virtual Educa 2018, 2018, Salvador. Virtual Educa 2018. México DF: Universidad Nacional Autónoma de México (UNAM), 2018b.

JENKINS, H. Cultura Convergente. Milano: Apogeo, 2007.

LUDKE, M.; ANDRÉ, M. E. D. A. Pesquisa em educação: abordagens qualitativas. São Paulo: Editora Pedagógica e Universitária,1986.

RIBBLE, M. S.; BAILEY, G.D.; ROSS, T.W. Digital Citizenship, addressing appropriate technology behavior. Learning \& Leading With Technology. Vol. 31, $\mathrm{n}^{\mathrm{o}}$ 01, 2014. Disponível em: http://www.digitalcitizenship.net/uploads/1stLL.pdf. Acessado em: 15 set. 2017.

RIFKIN, J. La tercera revolución industrial: como el poder lateral está transformando la energía, la economía y el mundo. Barcelona: Paidós, 2011.

MATTA, A.; SILVA, F.; BOAVENTURA, E. M. Design-based research ou pesquisa de desenvolvimento: metodologia para pesquisa aplicada de inovação em educação do século XXI. Revista da FAEEBA: Educação e Contemporaneidade, v. 23, p. 23-36, 2014.

MORIN, E. Introdução ao pensamento complexo. Porto Alegre: Sulina, 2005.

PENA, J. S. Mapa Bairros TBC Cabula. Correspondência por e-mail. Salvador, 25 de set de 2016.

SANTIAGO, R. C. C. A.; MATTA, A. Metacognição: construindo conhecimento sobre a metodologia Design-Based Research-DBR e sua utilização na educação a distância. $17^{\circ} \mathrm{CIAED}$ Congresso Internacional ABED de Educação a Distância, v. 1, p. 1-8, 2016. 
SESI. Relatório Executivo resultados 2018 - Janeiro a Dezembro. Diretoria de Educação e Tecnologia - DIRET - Unidade de Gestão Estratégica - UNIGEST. Confederação da Indústria, Brasília, 2018.

VYGOTSKY, Lev S. Linguagem, desenvolvimento e aprendizagem. São Paulo: Ícone, 2001.

Pensamento e Linguagem. Ed. 2a . São Paulo: Martins Fontes, 1998a.

. A Formação Social da Mente. 7. ed. São Paulo: Martins Fontes, 2007.

Teoria e Método em Psicologia. 3. ed. São Paulo: Martins Fontes. 2004

Recebido em: setembro de 2019.

Aprovado em: outubro de 2019. 